\title{
ON THE METAL AVERSION OF LGRBS
}

\author{
J.F. Graham ${ }^{1,2}$ and A.S. Fruchter ${ }^{1}$
}

\begin{abstract}
Recently, it has been suggested that the metallicity aversion of long-duration gamma-ray bursts (LGRBs) is not intrinsic to their formation, but rather a consequence of the anti-correlation between star-formation and metallicity seen in the general galaxy population. To investigate this proposal, we compare the metallicity of the hosts of LGRBs, broad-lined Type Ic (Ic-bl) supernovae (SNe), and Type II $\mathrm{SNe}$ to each other and to the metallicity distribution of star-forming galaxies using the SDSS to represent galaxies in the local universe and the TKRS for galaxies at intermediate redshifts.

The differing metallicity distributions of the LGRB hosts and the starformation in local galaxies forces us to conclude that the low-metallicity preference of LGRBs is not primarily driven by the anti-correlation between star-formation and metallicity, but rather must be overwhelmingly due to the astrophysics of the LGRBs themselves. Three quarters of our LGRB sample are found at metallicities below $12+\log (\mathrm{O} / \mathrm{H})<$ 8.6 , while less than a tenth of local star-formation is at similarly low metallicities. However, our SN samples are statistically consistent with the metallicity distribution of the general galaxy population. Using the TKRS population of galaxies, we are able to exclude the possibility that the LGRB host metallicity aversion is caused by the decrease in galaxy metallicity with redshift. The presence of the strong metallicity difference between LGRBs and Ic-bl SNe largely eliminates the possibility that the observed LGRB metallicity bias is a byproduct of a difference in the initial mass functions of the galaxy populations. Rather, metallicity below half-solar must be a fundamental component of the evolutionary process that separates LGRBs from the vast majority of Ic-bl SNe and from the bulk of local star-formation.
\end{abstract}

\footnotetext{
${ }^{1}$ Space Telescope Science Institute, 3700 San Martin Drive, Baltimore, MD 21218, USA

2 Department of Physics and Astronomy, Johns Hopkins University, Baltimore, MD 21218, USA
} 


\section{Introduction}

We compare the metallicity distribution of the hosts of Long-duration Gamma Ray Bursts (LGRB) with that of several other related populations: Type II supernovae (SNe) (which can serve as markers of star-formation), broad-lined Type Ic (Ic-bl) SNe (the specific type of SNe found coincident with LGRB events), and the general star-forming galaxy population. We perform this comparison across these different populations not primarily by number of galaxies but rather by weighting by their star-formation rates (SFRs). Our results show that, not only are the hosts of LGRBs at lower metallicities than either SNe hosts or general star-forming galaxies, but also that while Type Ic-bl and Type II SNe track star-formation (within our statistical ability to measure), more then three-quarters of our LGRBs are clustered in the lowest metallicity tenth of the star-formation.

To this end, we have compiled all spectra for LGRB and Type Ic-bl SNe hosts with host emission spectroscopy sufficient to allow metallicity measurement. To relate these events to the general star-forming galaxy population we have extracted the (approximately 137,000) galaxies from the Sloan Digital Sky Survey (SDSS) with line measurements suitable for metallicity measurement. This has also allowed us to compile a sample of the more common Type II SNe hosts simply by selecting the galaxies within our existing volume-limited SDSS sample which are known to have hosted such Type II SNe events with all the expected advantages of inter-sample consistency. Additionally, in order to better match the redshift range of our LGRB population, we extend our general star-forming galaxy population out to a redshift range of approximately unity via the addition of the higher redshift Team Keck Redshift Survey (TKRS) galaxy population.

Our analyses are based on four physical properties redshift, metallicity, rest frame B-band absolute magnitude, and (for most of our galaxies) the SFR. To maximize inter-sample consistency we have calculated independent metallicities using a common metallicity diagnostic, scale, and code via the $\mathrm{R}_{23}$ method implementation of Kobulnicky \& Kewley (2004). $R_{23}$ uses the ratio of oxygen to hydrogen line strengths (and the ratio of two oxygen lines to characterize the degree of ionization) to estimate the oxygen abundance in HII regions as a proxy of the total galaxy metallicity. This method however has a metallicity degeneracy issue where metal line emission cools the electron temperature causing the oxygen line strength, that was originally increasing with metallicity, to subsequently decrease yielding undifferentiable oxygen line strengths for both a high and low metallicity value. To resolve this degeneracy we have used observations of the $[\mathrm{N} \mathrm{II}] / \mathrm{H} \alpha$ line ratio, itself a crude metallicity indicator, to select between the upper and lower metallicity branch. For a few Type Ic-bl SNe we are forced to rely on this $[\mathrm{N} \mathrm{II}] / \mathrm{H} \alpha$ diagnostic exclusively with a corresponding increase in error. When comparing galaxies we use the B-band galaxy luminosity rather than galaxy mass as reliable host mass estimates were not available for a substantial fraction of the objects in our samples. We determine the SFRs from the galaxy's $\mathrm{H} \alpha$ emission via the Kennicutt (1998) metric. The primary difficulty with this is adjusting the $\mathrm{H} \alpha$ line flux for slit and fiber losses. 


\section{Results}

As shown in Modjaz et al. (2008) the low metallicity bias of LGRBs is visually apparent in a simple scatterplot of host metallicity versus absolute magnitude, when compared to that of the hosts of Type Ic-bl SNe and the general star-forming galaxy population, represented by the SDSS. This result becomes even more impressive with the approximate three fold increase the the number of LGRBs and a $50 \%$ increase in number of Type Ic-bl SNe presented in Figure 1. In this figure, we also add a second general star-forming galaxy population, the TKRS, to better reflect the higher redshift distribution of the LGRBs. In our first presentation of the scatter plot, we present the metallicity measured at the location of the LGRBs and SNe within their hosts to show the actual metallicity of the environment responsible for their creation. In later plots, however, we switch to plotting the host galaxy central metallicities in order to better compare with the general galaxy population. To obtain a fair sample of the galaxy population, we use a subset of the SDSS star-forming population restricted to a volume-limited sample. Even this population, however, does not represent the way we expect SNe or LGRBs to choose their hosts. Their probability of going off in a particular galaxy should be proportional to the rate of star-formation in that galaxy (all other things being equal). To better emulate the expected occurrence of LGRBs and SNe, we then further select among these galaxies via random selection weighted by the underlying star-formation of each galaxy. These volume-limited and star-formation weighted samples are plotted along with the similarly volume-limited Type II SNe in Figure 2.

As can be seen in Figure 2, the distribution of the star-formation weighted sample is significantly different from the distribution of galaxies by number with a large bias towards brighter galaxies having a greater fraction of star-formation. As the LGRBs in our sample extend to a redshift of about one, far beyond the reach of the SDSS, let alone our smaller volume-limited survey, we must consider the evolution of metallicity with redshift as a complicating factor. However the effect of abundance evolution over the redshift range of our sample is nowhere near as dramatic as the metal aversion of the LGRB population as a whole. While there are three LGRB hosts in the high metallicity range of our sample (LGRBs 051022, 020819B, \& 050826), is it clearly apparent in all three metallicity vs. luminosity scatterplots (Figs. 1 \& 2) that while the SNe are distributed with the general star-forming galaxy population the LGRBs are on whole at the bottom of the metallicity distribution. It is notable that the three high metallicity (out of 14 total) LGRBs do appear consistent with the general star-forming galaxy population of comparable brightness and redshift. This is intrinsically surprising as were the metal aversion to remain in effect for these objects we would expect their occurrence, if still in the high metallicity range, to be far lower then the typical metallicity for the population at that luminosity and redshift (i.e. either an outlier of said population or among the lowest galaxies available within it).

While the metallicity vs. luminosity scatterplots show quite conclusively that the hosts of LGRBs are at lower metallicites than either SNe hosts or general 


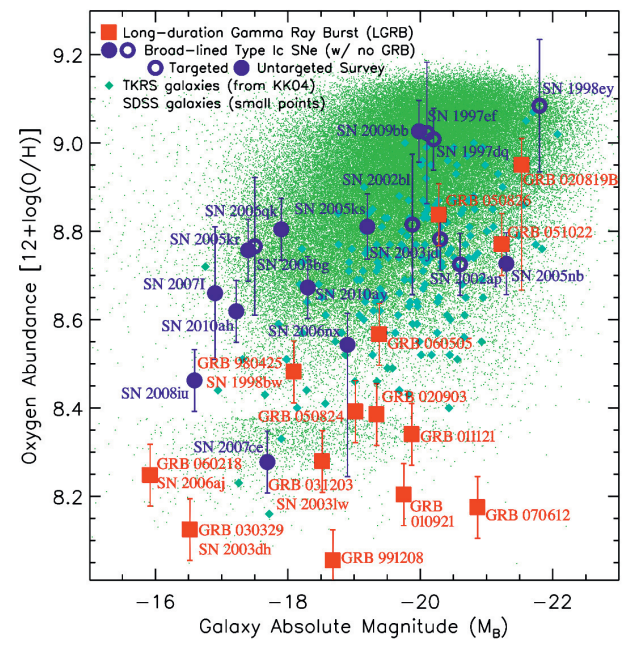

Fig. 1. Site Metallicity vs. absolute B-band galaxy luminosity of LGRB (squares) and broad-lined Type Ic SNe hosts (circles). Filled circles represent $\mathrm{SNe}$ selected in an untaragted manner whereas open circlers are from galaxy targeted SNe surveys (and thus may be biased in galaxy properties by target selection). Star-forming galaxies from the SDSS (small dots) and TKRS (diamonds) are plotted in the background to provide a low and high redshift comparison sample respectively. Note the profound difference between the LGRB metallicity values and those of the Type Ic-bl SNe.

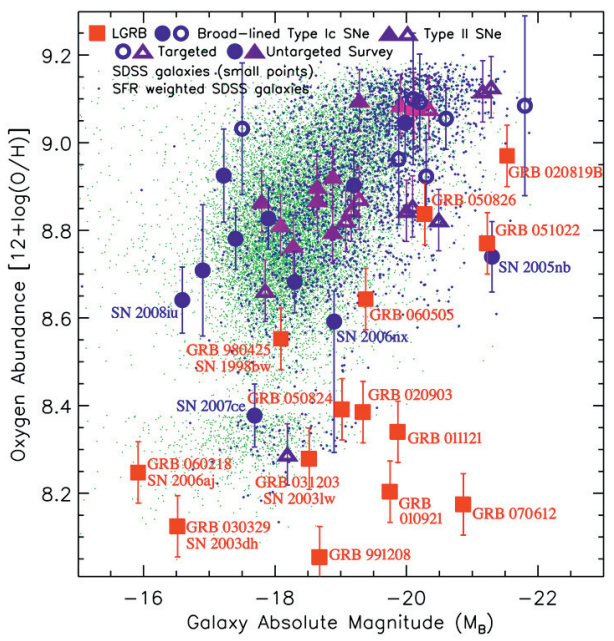

Fig. 2. Central Metallicity vs. B-band absolute galaxy luminosity. The Type II SNe hosts (purple triangles), SDSS galaxies (small green points) and the star-formation weighted random SDSS galaxies synthetic population (small blue points) shown here are limited to the sample redshift range of $0.02<z<0.04$. In order to get reasonable comparison samples, no redshift cut has been applied to the Type Ic-bl host population or the LGRB population. Note that the star-formation weighted random SDSS galaxy population tracks the SNe quite well in luminosity-metallicity space.

star-forming galaxies, they do not directly address whether this metallicity bias could be caused by the anti-correlation between SFR and metallicity of Mannucci et al. (2010) as claimed in Mannucci et al. (2011). To confront this issue, we plot in Figure 3 the integrated star-formation of the SDSS sample as a function of metallicity in comparison with the cumulative distributions of LGRBs and SNe. A similar plot, with a much smaller LGRB sample and no comparison of SNe, was first shown by Stanek et al. (2006). Both the non-LGRB Type Ic-bl SNe and Type II SNe track the distribution of star-forming SDSS galaxies quite well. The LGRBs however display a profound preference for lower metallicities. Thus the CCSNe track star-formation independent of its metallicity, while LGRBs do not.

However, this result could still be consistent with the Mannucci et al. (2010) relation, if the SFRs of the LGRB host were wildly discrepant from the other populations. To exclude this possibility, in Figure 4 we directly compare the SFRs of the LGRB and SNe hosts to the general star-forming SDSS galaxy population. 


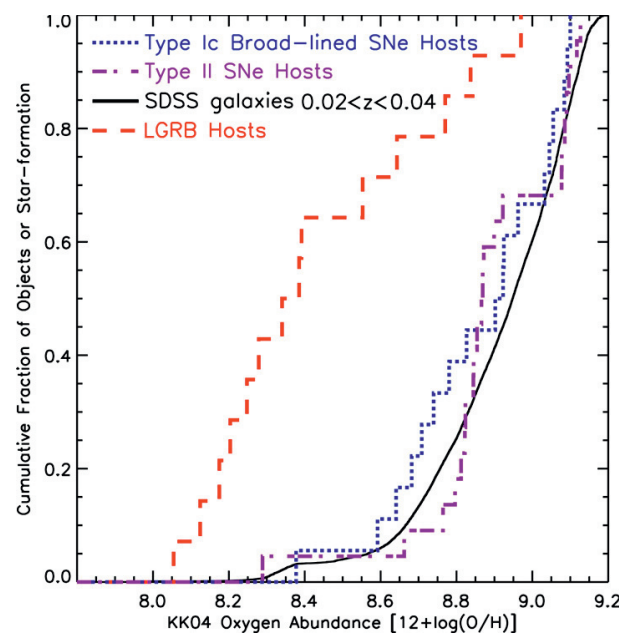

Fig. 3. Cumulative fraction of population (LGRBs and $\mathrm{SNe}$ ) or total star-formation (redshift-cut SDSS star-forming sample) vs. galaxy central metallicity. Only the SDSS galaxies, and the Type II SNe hosts (which are all included in the SDSSS sample), are limited to the redshift-cut range. The LGRB hosts have metallicities considerably lower than would be obtained simply by following star-formation while both types of supernovae are consistent with the star-formation distribution. Thus the metallicity distribution of LGRBs cannot be explained only by association with starforming galaxies.

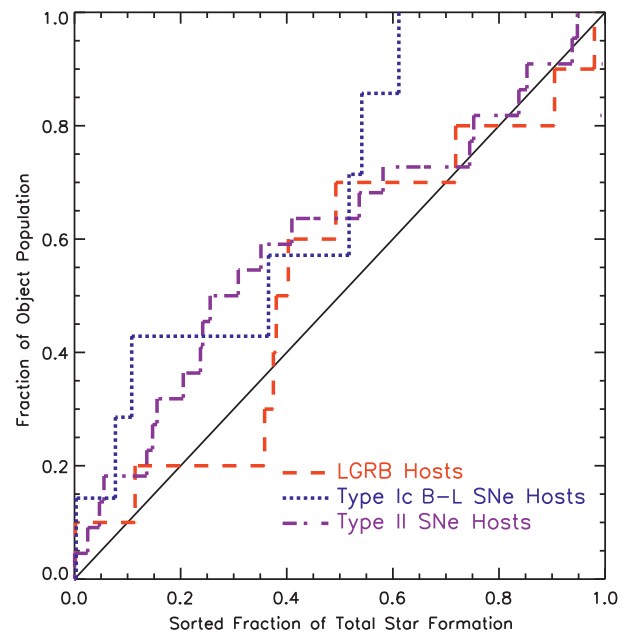

Fig. 4. Normalized cumulative distribution of star-formation fractional values for the SNe and LGRBs. For each object we determine the fraction of star-formation contained in SDSS galaxies of equal or lesser SFR than the object's host. All populations are reasonably consistent with the diagonal line indicating a reasonably consistent SFR distribution. Thus while LGRB hosts do have higher than average SFRs, as noted by Mannucci et al. (2011), their SFRs agree with what one would expect of a population that tracks star-formation. Thus metallicity, not SFR, must be the source of the discordant results shown in Figure 3.

This comparison is performed by taking the SFR of each LGRB and SNe host and determining the fraction of the total star-formation in the general SDSS galaxy population that occurs in galaxies with less star-formation than the host. These fractional values are then sorted and plotted as a normalized cumulative histogram. Should the distribution of star-formation for a given object type follow the general star-forming SDSS galaxy population then this histogram would track a diagonal line on the plot. For the SNe, the SDSS comparison sample is volume-limited. However, for the LGRBs, which are intrinsically a magnitude-limited sample, we use the entire SDSS, as a comparison magnitude-limited sample. Both the LGRBs and targeted Type II SNe population track the diagonal well, indicating a good correspondence between the SFRs of the two populations and the general SDSS galaxy population, and suggesting that SFR is correlated with LGRB and SNe formation. 


\section{Conclusions}

We have shown quite conclusively in various scatterplots that the hosts of LGRBs are at lower metallicites than either SNe hosts or general star-forming galaxies. However, in order to directly address the question posed by Mannucci et al. (2011), of whether SFR can explain metallicity, we plot as a function of metallicity the integrated star-formation of the SDSS sample in comparison with the cumulative distributions of LGRBs and SNe. There we find that three quarters of our LGRBs are found in the in the bottom low-metallicity tenth of the star-formation, with the remaining quarter (like our SNe populations) appearing to track starformation independent of metallicity. Assuming, all else being equal, that LGRBs (and SNe) occur proportionally to the allowable star-formation this bias indicates that LGRBs clearly prefer much lower metallicity host environments (as first suggested in Stanek et al. 2006 and shown with a much larger sample here). We also consider the SFR distribution of the LGRB and SNe populations and find that they are consistent with the SFR distribution of the SDSS sample and especially consistent if this comparison is limited only to SDSS objects of similar metallicity. Interestingly we find that the SFR distribution of the low-metallicity SDSS galaxies is reasonably similar to that of the entire SDSS population and cannot determine which the LGRB population best tracks.

In contrast to LGRBs, both our Type II and Type Ic-bl SNe populations appear to track the metallicity of the integrated SDSS star-formation. This is what one would expect if star-formation alone is sufficient to explain the metallicity distribution of both SNe types. Nonetheless, any metallicity bias, even one much more minor than that seen with LGRBs, has significant implications for a unified standard formation model for Type Ic SNe, Type Ic-bl SNe, and LGRBs. Kelly \& Kirshner (2012) and Sanders et al. (2012) have looked at the metallicity distribution of different SNe types vs. each other (without relation to the SDSS or LGRBs) and highlight a bias towards Type Ic-bl SNe preferentially occurring in slightly lower metallicities than the other SNe populations (including the non-bl Type Ic SNe). Due to our strict volume limits, imposed to allow comparison with the SDSS, our Type II SNe sample is far smaller than that used in these other works. And we have made a decision to only look at two SNe types - Type II SNe as a hopefully truly unbiased indicator of star-formation, and Type Ic-bl SNe, due to their close association with LGRBs. Thus, while our work is well suited for its primary purpose of determining whether the metallicity distribution of LGRB hosts differs from other star-forming galaxies, it is much less powerful for distinguishing relative metallicity preferences of SNe. However, the metallicity differences between Type Ic-bl and other SNe, hinted at in our sample and perhaps seen more clearly in these other works, is dwarfed by the strong metallicity bias seen in LGRBs.

The presence of a metallicity bias between LGRBs and Type Ic-bl SNe poses a problem for explanations of the LGRB metallicity bias being the incidental result of an IMF difference in their host galaxies. In nearly all cases where one would have expected to detect a Type Ic underlying a LGRB to have been detected, 
one has been found, and where good spectroscopy is available, the Type Ic is broad-lined (see Cano 2012 for a good discussion of this point). Thus, many and perhaps all Type Ic-bl SNe share a common progenitor type with LGRBs. One would therefore expect the masses of Type Ic-bl SNe progenitors to be similar to those of LGRBs. Thus while IMF differences between galaxies could play some role in determining where one finds LGRBs, the IMF is almost certainly far less important than galaxy metallicity.

These observations do not agree with the suggestion of Mannucci et al. (2011) "that the difference with the mass-metallicity relation is due to higher than average SFRs [of LGRB hosts] and that LGRBs with optical afterglows do not preferentially select low-metallicity hosts among the star-forming galaxies." While the average SFR of LGRB hosts is indeed higher than that of typical SDSS galaxies, this is because LGRBs do not choose galaxies based on number but rather based on SFR (as well as metallicity). The star-formation distribution of the LGRB host population tracks that of similar metallicity SDSS galaxies, Indeed, due to the fact that the star-formation distribution of galaxies in the SDSS is largely independent of metallicity, they track the star-formation distribution of the entire SDSS as best as can be determined with only the 11 LGRBs for which we have good SFRs. The LGRB hosts population is explicitly concentrated in the low metallicity end of the available star-formation. While the LGRB hosts themselves may remain consistent with the mass, metallicity, and SFR relation of Mannucci et al. (2010), this relationship is not sufficient to explain the observed concentration of LGRBs in low metallicity star-formation.

Nonetheless, the preference of LGRBs for low-metallicity hosts is not absolute. Three cases of LGRBs in roughly solar metallicity hosts (LGRBs 051022, 020819B, \& 050826) demonstrate that LGRBs can occur at high metallicity. However, such events are quite rare. These high-metallicity objects are all at substantial redshifts ( $99 \%$ of the galaxies in the SDSS sample lie closer than the closest of these LGRBs), and thus the search volume needed to find them was large. As a result, our sample overemphasizes high-metallicity LGRBs compared to the distribution likely to be found in a volume-limited survey. The presence of these objects does not substantially detract from our main conclusion, that on the whole, LGRBs have a strong intrinsic preference for low metallicity environments.

\section{References}

Cano, Z., 2012 [arXiv:1208.0307]

Kelly, P.L., \& Kirshner, R.P., 2012, ApJ, 759, 107

Kennicutt, R.C., Jr., 1998, ARA\&A, 36, 189

Kobulnicky, H.A., \& Kewley, L.J., 2004, ApJ, 617, 240

Mannucci, F., Cresci, G., Maiolino, R., et al., 2010, MSRAS, 408, 2115

Mannucci, F., Salvaterra, R., \& Campisi, M.A., 2011, MSRAS, 414, 1263

Modjaz, M., Kewley, L., Kirshner, R.P., et al., 2008, AJ, 135, 1136

Sanders, N.E., Soderberg, A.M., Levesque, E.M., et al., 2012, ApJ, 758, 132

Stanek, K.Z., Gnedin, O.Y., Beacom, J.F., et al., 2006, Acta Astronomica, 56, 333 
